\title{
GMR
}

\section{Direct preparation protocol to obtain mitotic chromosomes from canine mammary tumors}

\author{
C.S.D. Morais ${ }^{1}$, P.R.A.M. Affonso ${ }^{2}$, J.A. Bitencourt ${ }^{2}$ and A.A. Wenceslau \\ 'Departamento de Ciências Agrárias e Ambientais, \\ Programa de Pós-Graduação em Ciência Animal, Illhéus, BA, Brasil \\ ${ }^{2}$ Departamento de Biologia, Programa de Pós-Graduação em Genética, \\ Biodiversidade e Conservação, Jequié, BA, Brasil \\ Corresponding author: C.S.D. Morais \\ E-mail: cimovet@yahoo.com.br
}

Genet. Mol. Res. 14 (4): 19389-19395 (2015)

Received July 28, 2015

Accepted October 9, 2015

Published December 29, 2015

DOI http://dx.doi.org/10.4238/2015.December.29.49

\begin{abstract}
Currently, mammary neoplasms in female canines are a serious problem in veterinary clinics. In addition, the canine species is an excellent disease model for human oncology because of the biological and genetic similarities between the species. Cytogenetics has allowed further study of the characterization of neoplasms in canines. We hypothesized that the use of a direct preparation protocol for mitotic chromosome analysis would provide a simple and low cost protocol for use in all laboratories. The objective of this method is to display in a few hours of dividing cells just like the time of collection since cell division in tissue can be obtained. Ten female canines with the spontaneous occurrence of mammary neoplasia were used to test a pioneering direct preparation protocol to obtain mitotic chromosomes. The excised breast tumor tissue fragments were subjected to the protocol consisting of treatment with colchicine, treatment with hypotonic solution, and fixation. Mitotic chromosomes were absent in cell suspensions of only two samples among the 10 materials analyzed, based on the analysis of five blades for each preparation obtained. So, the cell suspension obtained allowed for the observation of eight tissue samples viable for cytogenetic analysis, five of which had excellent numbers of mitotic
\end{abstract}


chromosomes. However, the technique was unsuccessful in producing high-quality cell suspensions because of inadequate condensation and scattering of chromosomes. While adjustments to methodological procedures are needed, this protocol represents a low cost and simplified method to study the cytogenetics of canine tumors.

Key words: Chromosome alterations; Breast cancer; Canine; Cytogenetics; Neoplasm

\section{INTRODUCTION}

Neoplasms in dogs represent an important clinical problem, especially owing to the increased longevity of these animals by advances in veterinary medicine (Matos et al., 2012). Among all types of neoplasms, those affecting the mammary glands occur with a high incidence and target both sexually intact and spayed female dogs (Cassali et al., 2011). Dogs naturally develop neoplasms (Rowell et al., 2011) that are characteristically similar with some human tumors, making them excellent models for study because of the epidemiological, clinical, biological and genetic similarities between the species (Khanna et al., 2006; Arley, 2012).

There is great interest in expanding the work on chromosomal changes that occur in canine tumor cells because these studies can offer benefits to human disease as well (ReimannBerg et al., 2012). In this sense, the contribution of cytogenetics to the identification of chromosomal changes that are associated with the occurrence of tumors promotes the improvement of diagnostic and prognostic techniques (Sumner, 2003; Sargan et al., 2005; Breen, 2008; Devitt et al., 2009; Ferguson-Smith, 2013). The initial step for chromosomal studies is to obtain cells in metaphase because during this phase of the cell cycle, chromosomes are condensed and individualized and can be viewed through an optical microscope. The cell suspension can be obtained with different methodologies that have similar steps, including obtaining tissues with cells in division, treatment of the tissues with colchicine for disruption of cells in metaphase, and treatment of the tissues with a hypotonic solution for spreading of the cells and chromosomes (Hare et al., 1966; Kasahara, 2009). Considering these characteristics, it is understandable that a large amount of information on leukemia and hematopoietic cancers and neoplasms has been obtained through cytogenetics since it is easier to obtain good chromosome preparations from blood cells than from solid tissue (Sumner, 2003). The solid tumors usually present with a low mitotic index and require long-term cell culture and methodologically complex processes to visualize chromosomes. In addition, these tissues are capable of generating a lineage bearer of chromosomal changes that do not reflect the constitution of the individual tumor karyotype (Sandberg, 1988). Despite these limitations, short or long term cell cultivation is still the most commonly used technique in obtaining chromosomes from solid tumors (Sargan et al., 2005; Devitt et al., 2009; Reimann-Berg et al., 2012).

Cell cultivation techniques rely on specialized equipment, including laminar flow for maintaining an aseptic environment and an inverted microscope for monitoring the multiplication of cells in culture. Thus, this cannot be regarded as a routine method since few diagnostic laboratories and clinics are properly equipped for these techniques, especially in developing countries. In addition, cell culture lacks standardization of cultivation techniques and often results in the heavy consumption of consumables in order to obtain a reliable and reproducible in vitro system (Kasahara, 2009). As an alternative method, the direct preparation technique is a simple 
and quick way of obtaining chromosome preparations and is widely used in cytogenetic studies of insects (Webb et al., 1978), fish (Netto et al., 2007), amphibians (Bogart, 1973), reptiles (King and Rofe, 1976), birds (Shoffner et al., 1967) and small mammals (Ford and Harmerton, 1956). In this method, dividing cells can be obtained in just a few hours for cytogenetic analysis (Hare et al., 1966; Kasahara, 2009). While neoplastic cells continue to divide (Kusewitt and Rush, 2007), the standardization of a direct method for obtaining chromosome preparations of solid neoplasms in dogs is hypothetically possible. We have used this methodology for the first time in the preparation of tissues for direct canine mammary tumor (CMT) cytogenetics as an alternative method to routine cytogenetics in dogs.

\section{MATERIAL AND METHODS}

\section{General methods}

Materials were collected at the Veterinary Hospital of the State University of Santa Cruz in Ilhéus, Brazil and in the Animal Health Veterinary Clinic in the town of Jequié, Brazil. The histopathological analysis was performed in the laboratory of pathological anatomy in the Hospital of Veterinary Medicine of the University Federal da Bahia. The analysis of the material for the cytogenetic study was performed in the Cytogenetic Laboratory in the Department of Biological Sciences of the Universidade Estadual do Sudoeste da Bahia at the Jequié campus.

\section{Animals}

Female canines $(\mathrm{N}=10)$ with spontaneous neoplasia were used for gathering material between January and July 2012. The female canines were clinically diagnosed as carriers of mammary neoplasia and received surgical indication for removal of the tumor or tumors as part of the treatment protocol. The animals had all clinical and laboratory examinations as a pre-surgery requirement. Information on the health history and neoplasm diagnosis of the animals was obtained at the time of tissue collection.

\section{Tumor collection and cytogenetic analysis preparation}

Removal of the breast tumor or tumors was performed by a surgical procedure, using the proper technique according to the physical appearance of neoplasia. Three to four pieces of the tumor (approximately $1 \times 1 \times 1 \mathrm{~cm}$ ) were collected from each tumor mass and conditioned in RPMI 1640 culture medium at $4^{\circ} \mathrm{C}$. The material was kept refrigerated until arrival at cytogenetics laboratory for at most 1 hour.

\section{Histopathological analysis}

For histopathological characterization, tumor tissue fragments were collected and fixed in formalin solution immediately after the surgical removal of the tumor. After fixation, the material was included in paraffin, the tumor pieces were sliced into sections by using a microtome, mounted on blades, and stained with hematoxylin-eosin. Following this, they were examined by a light microscope. 


\section{Mitotic chromosome retrieval from tumor cells}

The technique used to obtain mitotic chromosomes from tumor cells was described by Netto et al. (2007) and all modifications are described below. Tumor fragments were mechanically fractionated with scissors, a surgical blade, and rat tooth forceps. The resulting solution was filtered in gauze for removal of larger fragments. This solution was transferred to a $15-\mathrm{mL}$ conical tube and RPMI 1640 culture medium was added to bring the volume to $9.5 \mathrm{~mL}$. Five drops of colchicine $(0.05 \%)$ were added to the tube and the solution was homogenized with a Pasteur pipette at $38^{\circ} \mathrm{C}$ for $30 \mathrm{~min}$. Subsequently, the material was centrifuged at $1000 \mathrm{rpm}$ for $10 \mathrm{~min}$. The supernatant was discarded and the pellet was resuspended in $10 \mathrm{~mL}$ hypotonic solution $(0.075 \mathrm{M} \mathrm{KCl})$. After 20 minutes of incubation at $38^{\circ} \mathrm{C}$, five drops of Carnoy fixative solution (in $3: 1$ methanol to acetic acid) was added on ice and the solution was homogenized again. After this, the suspension was centrifuged for $10 \mathrm{~min}$ at $1000 \mathrm{rpm}$. The supernatant was discarded and $8 \mathrm{~mL}$ fixative Carnoy solution (in 3:1 methanol to acetic acid) was added again. Following this, the suspension was homogenized, centrifuged for $10 \mathrm{~min}$ at $1500 \mathrm{rpm}$. Addition of Carnoy solution, homogenization, and centrifugation were repeated 3 times to remove cellular debris. After the last centrifugation, 2 $\mathrm{mL}$ fixative solution was added and, after homogenization, the cell suspension was transferred to $1.5-\mathrm{mL}$ tubes for storage at $-20^{\circ} \mathrm{C}$.

\section{Preparation of blades and analysis of metaphase}

The blades used for microscopy were washed and stored in $70 \%$ ethanol. Before use, the blades were air-dried (Rothfels and Siminovitch, 1958). The chromosomes in mitotic metaphase from the neoplastic tissue fragments were stained by the May-Grunwald-Giemsa method (Vallada, 1999) using Giemsa-dye diluted 10:1 in phosphate buffer (pH 6.8) and incubated for 10 min, to establish the number and karyotype of formula diploid in each sample. After washing with distilled water and air drying, samples were analyzed under an optical microscope Olympus BX51 (Tokyo, Japan) 1000X; with a digital image capturing system Image ProPlus 6.2; Media Cybernetics (Maryland, USA).

\section{RESULTS}

In the histopathological analysis, more than one histological classification was identified in five individuals (Table 1 ) and multiple samples were collected from individual 1 because there were different tumor masses in the animal. Mitotic chromosomes were absent in the cell suspensions of only three individuals among the 10 analyzed. The histological classifications of samples of individuals 7 and 9 that did not allow their respective analyses as cells from exclusively of CMT. Therefore, the cell suspensions of these individuals were considered inadequate for cytogenetic analysis of CMT neoplastic cells.

In most individuals, at least 30 chromosomes in mitotic metaphase were observed; in some cases, this number was obtained after viewing the same chromosome preparation at least 10 times. Individuals $1,5,6,8$, and 10 gave the best results with respect to mitotic chromosomes and individuals 2,3 , and 7 averaged only 1 metaphasic cell per blade (Table 1). It was possible to observe individualization and condensation of the chromosomes in metaphase between different cellular suspension spreading patterns (Figure 1). Concerning the quality of cell suspensions, 
several individuals presented insufficient mitotic chromosome spreading and chromosome overlap was frequently observed. In addition, it was possible to identify different degrees of condensation of the chromosomes between the materials, some of which were more distended and others more condensed.

Table 1. Classification of histological samples of dogs with canine mamary tumors and the number of metaphasic cells in each sample after cytogenetic preparation.

\begin{tabular}{llr}
\hline Sample & Histological classification & Number of cells in metaphase \\
\hline Individual 1-sample 1 & Complex carcinoma & 36 \\
Individual 1-sample 2 & Carcinoma mixed tumor and ductal papilloma & 0 \\
Individual 2 & Papillary carcinoma & 13 \\
Individual 3 & Papillary carcinoma and ductal papillary carcinoma & 10 \\
Individual 4 & Mixed tumor carcinoma & 0 \\
Individual 5 & Mixed tumor carcinoma & 30 \\
Individual 6 & Papillary adenoma, papillary-tubule adenoma and papillary & 30 \\
& adenoma with focal area of transformation carcinomatosum & 10 \\
Individual 7 & Papillary carcinoma, lobular hyperplasia and benign mixed tumor & 30 \\
Individual 8 & Papillary carcinoma & 0 \\
Individual 9 & Lactating mammary gland mixed tumor carcinoma and papillary carcinoma & 105 \\
Individual 10 & Carcinosarcoma & \\
\hline
\end{tabular}

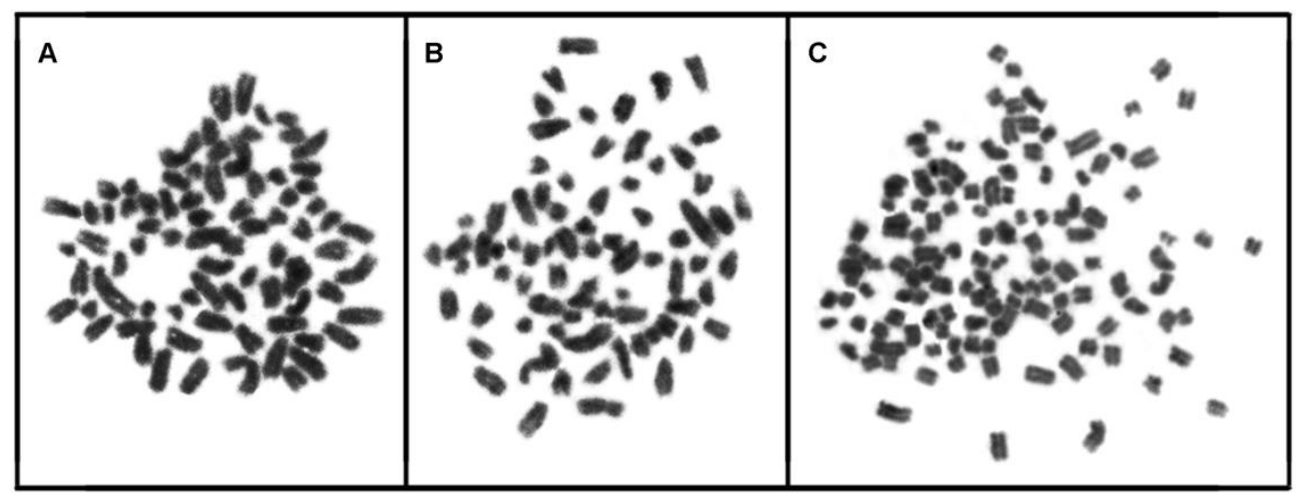

Figure 1. Visualization of cells in metaphase obtained by direct preparation of canine mammary tumors. A. Papillary adenoma, papillary-tubule adenoma $(2 n=80)$. B. Papillary carcinoma and ductal papillary carcinoma $(2 n=77)$. C. Carcinosarcoma $(2 n=129)$.

\section{DISCUSSION}

The existence of more than one histological type in different parts of the same node or on different tumors of the same animal can occur in CMT (Cassali et al., 2011), and this was observed in half the cases studied in this work. Considering this possibility, it is preferable to individually package the tumor fragments from CMT for cytogenetic evaluation to avoid collection errors and prevent cytogenetic preparations with different histological diagnoses in the same cell suspension (this was the case for individuals 7 and 9). However, different classifications of CMT within the same histological sample are not always feasible because these diagnostic differences can be due to the structural organization of the tissue, and not its cellular or histological characterization (this was the case for individuals 3 and 6). 
The difficulty in observing metaphase in a cell suspension from solid tumors using direct preparation may not only be owing to the low mitotic index (Sandberg, 1988), but also the dissociation of the solid tissue in the culture medium. Inadequate tissue dissociation can form aggregate cell that can mask the presence of mitotic metaphase. A solution for this problem would be treating the tissue with the enzyme collagenase prior to cell cultivation (Reimann-Berg et al., 2012), which should provide improved cell breakdown and increase the chances of viewing mitotic chromosomes.

The number of mitotic metaphases analyzed for characterization of the karyotype of tumors varies between cytogenetic studies (Mayr et al., 1990; Reimann et al., 1999; Winkler et al., 2005; Winkler et al., 2006); however, at least 30 cells in metaphase are frequently identified. In our study, we observed that half of the individuals studied had less than 30 metaphasic cells in 10 slides analyzed, indicating that cell suspensions obtained with the direct preparation technique can provide a satisfactory number of mitotic metaphases for study of CMT neoplastic cells.

While the treatment of tissue with colchicine, hypotonic solution, and fixing solution are fundamental steps in obtaining mitotic chromosomes of good quality for karyotype analysis, several factors can influence the results of cytogenetics, including cell type and species studied, and standardized technical protocols and methods need to be adopted in each laboratory (Kasahara, 2009). In this study, we used the same protocol for all individuals in order to test its use as a ubiquitous method of cytogenetic analysis for CMT.

Results of individualization and condensation of the chromosomes in metaphase between different cellular suspension spreading patterns corroborate the observations of Netto et al. (2007), which, in a cytogenetic study of fish, show that variations in quality of mitotic metaphase may require the development or adaptation of techniques for the species studied. In the case of CMT, it is suggested to test different concentrations of colchicine solution and treatment times with hypotonic solution in the same tumor tissue in order to obtain better results. Another harmful factor in the spreading and individualization of chromosomes is the presence of cytoplasmic residue in the cell suspension (Kasahara, 2009). Potential solutions to this problem are multiple washes, the use of products that remove cytoplasmic content during fixation like Cytoclear (Genial Genetics Solutions Ltd, UK) and the appropriate dilution of the solutions. These modifications can lead to improvement in the quality of cytological preparations, since solid tissue preparations have a high degree of cellularity.

It is worth noting that the use of this technique should allow for consistent use of cytogenetic studies in canine oncology because of the simplicity of the facilities and equipment necessary for its completion. The use of the direct preparation protocol to obtain mitotic chromosomes from neoplastic tissue from canine mammary glands is a simple and inexpensive technique, although necessary changes are needed to improve the methodological procedures tested in this study. Therefore, this pioneering work using cytogenetics shows that researchers of canine oncology can adopt this methodology, as studies with solid tumors in dogs will provide more information on chromosomal changes in canine neoplasms. This will not only benefit these animals but will also provide additional information for comparative studies with human cancer cytogenetics.

Our study shows that the direct preparation protocol to obtain mitotic chromosomes from canine mammary tumors can be used to study canine tumors by cytogenetics in laboratories that are minimally equipped and at a low cost. However, changes to the methodological procedures tested here are still required to increase the quality of the spreading of chromosomes. 


\section{Conflicts of interest}

The authors declare no conflict of interest.

\section{ACKNOWLEDGMENTS}

This work is part of the dissertation of C.S.D. Morais, at the Graduate Program in Animal Science from the State University of Santa Cruz, Bahia. Thanks to FAPESB for granting the scholarship and veterinary Camila Calheira, owner of Animal Health Veterinary Clinic, Jequié.

\section{REFERENCES}

Arley R (2012). Lab reports and cat scans: can veterinary oncology guide our way to new treatments for human cancers? Future Med. Chem. 4: 1391-1394.

Bogart JP (1973). Method for obtaining chromosomes. Caldasia 11: 29-40.

Breen M (2008). Canine cytogenetics-from band to basepair. Cytogenet. Genome Res. 120: 50-60.

Cassali GD, Lavalle GE, De Nardi AB, Ferreira E, et al. (2011). Consensus for the diagnosis, prognosis and treatment of canine mammary tumors. Braz. J. Vet. Pathol. 4: 153-180.

Devitt JJ, Maranon DG, Ehrhart EJ, Bachand AM, et al. (2009). Correlations between numerical chromosomal aberrations in the tumor and peripheral blood in canine lymphoma. Cytogenet. Genome Res. 124: 12-18.

Ford C and Harmerton JL (1956). A colchicine, hypotonic citrate, squash sequence for mammalian chromosomes. Stain Technol. 31: 247-251.

Hare WCD, Weber WT, Mcfeely RA and Tsu-Ju Yang (1966). Cytogenetics in dog and cat. J. Small Anim. Pract. 7: $575-592$.

Kasahara S (2009). Introduction to research in cytogenetics of vertebrates. 1st edn. The Brazilian Society of Genetics, Ribeirão Preto.

Khanna C, Lindblad-Toh K, Vail D, London C, et al. (2006). The dog as a cancer model. Nat. Biotechnol. 24: 1065-1066.

King M and Rofe R (1976). Karyotypic variation in the Australian gekko Phyllodactylus marmoratus (Gekkonidae: Reptilia). Chomosoma 57: 75-87.

Kusewitt DF and Rush LJ (2007). Neoplasm and tumor biology. In: Foundations of pathology in veterinary medicine (Mcgavin MD and Zachary JF, eds.). Elsevier, Rio de Janeiro, 253-298.

Matos AJF, Baptista CS, Gaertner MF and Rutteman GR (2012). Prognostic studies of canine and feline mammary tumours: the need for standardized procedures. Vet. J. 193: 24-31.

Mayr B, Schleger W, Kalat M, Schweiger P, et al. (1990). Cytogenetic studies in the canine mammary tumor. Cancer Genet. Cytogenet. 47: 83-87.

Netto MRCB, Pauls E and Affonso PRM (2007). A standard protocol for obtaining fish chromosomes under post-mortem conditions. Micron 38: 214-217.

Reimann N, Nolte I, Bartnitzke S and Bullerdiek J (1999). Cancer genetics going to the dogs. J. Natl. Cancer Inst. 91: $1688-1689$.

Reimann-Berg N, Bullerdiek J, Murua Escobar H and Nolte I (2012). Chromosome analyses in dog. Tierarztl. Prax. Ausg. K Kleintiere Heimtiere 3: 191-196.

Rothfels KH and Siminovitch L (1958). An air-drying technique for flattening chromosomes in mammalian cells grown in vitro. Biotech. Histochem. 33: 73-77.

Rowell JL, McCarthy DO and Alvarez CE (2011). Dog models of naturally occurring cancer. Trends Mol. Med. 17: $380-388$.

Sandberg AA (1988). Chromosomes in Solid Tumors and Beyond. Cancer Res. 48: 1049-1059.

Sargan DR, Milne BS, Aguirre Hernandez J, O'Brien PCM, et al. (2005). Chromosome rearrangements in canine fibrosarcomas. J. Hered. 96: 766-773.

Shoffner RN, Krishan A, Haiden GJ, Bammi RK, et al. (1967). Avian chromosome methodology. Poult. Sci. 46: $333-344$.

Sumner AT (2003). Chromosomes: Organization and Function. 1st edn. Blackwell Publishing.

Vallada, EP (1999). Manual hematological techniques. 1st edn. Atheneu, São Paulo.

Webb AIR, White MJD, Contreras N and Cheney J (1978). Cytogenetics of the parthenogenetic grasshopper Warramaba (formerly Moraba) virgo and its bisexual relatives. Chromosoma 67: 309-339.

Winkler S, Murua Escobar H, Eberle N, Reimann-Berg N, et al. (2005). Establishment of a cell line derived from a canine prostate carcinoma with a highly rearranged karyotype. J. Hered. 96: 782-785.

Winkler S, Reimann-Berg N, Murua Escobar H, Loeschke S, et al. (2006). Polysomia 13 in a canine prostate carcinoma underlining its significance in the development of prostate cancer. Cancer Genet. Cytogenet. 169: 154-158. 